UCRL-CR-125917

B317413

Influence of Crushing and Additive Irradiation Procedures on EPR Dosimetry of Tooth Enamel

S.V. Shalom, V.V. Chumak, E.H. Haskell, R.B. Hayes, G.H. Kenner

January 1996

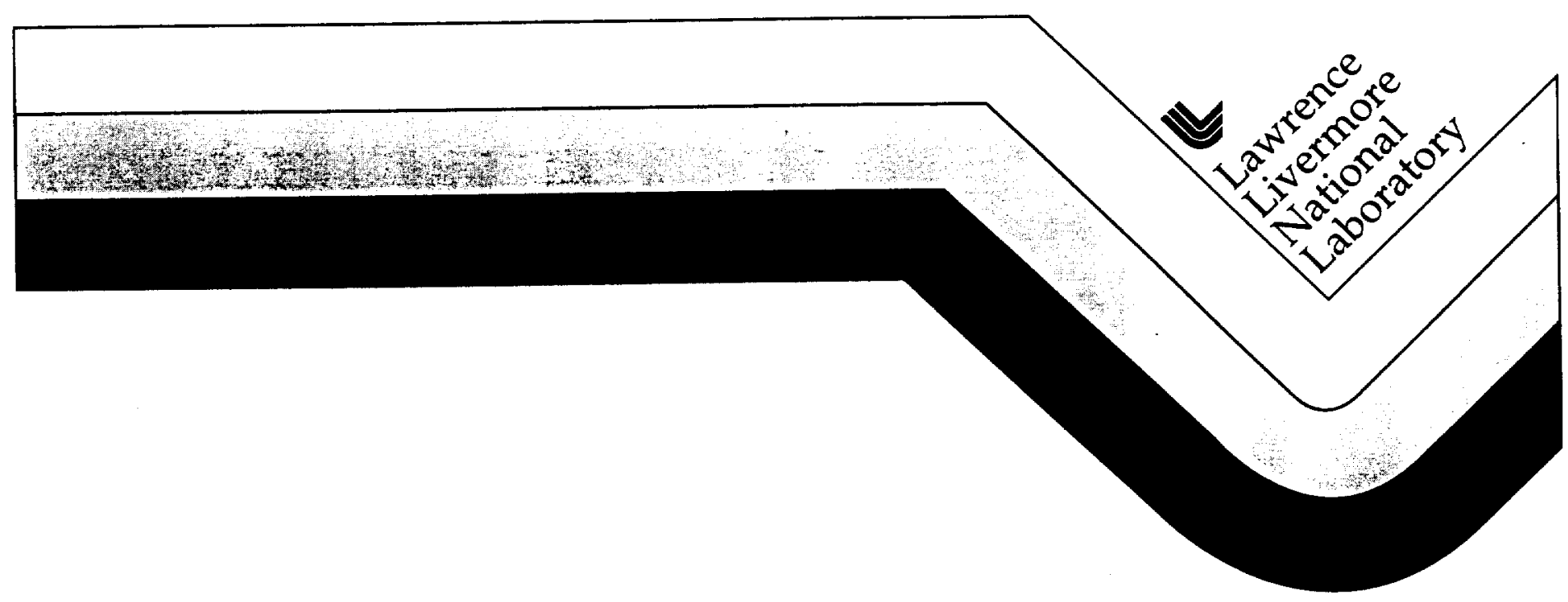




\section{DISCLAIMER}

This document was prepared as an account of work sponsored by an agency of the United States Government. Neither the United States Government nor the University of California nor any of their employees, makes any warranty, express or implied, or assumes any legal liability or responsibility for the accuracy, completeness, or usefulness of any information, apparatus, product, or process disclosed, or represents that its use would not infringe privately owned rights. Reference herein to any specific commercial product, process, or service by trade name, trademark, manufacturer, or otherwise, does not necessarily constitute or imply its endorsement, recommendation, or favoring by the United States Government or the University of California. The views and opinions of authors expressed herein do not necessarily state or reflect those of the United States Government or the University of California, and shall not be used for advertising or product endorsement purposes.

Work performed under the auspices of the U.S. Department of Energy by Lawrence Livermore National Laboratory under Contract W-7405-ENG-48. 


\title{
INFLUENCE OF CRUSHING AND ADDITIVE IRRADIATION PROCEDURES ON EPR DOSIMETRY OF TOOTH ENAMEL
}

\author{
S.V. Sholom*, E.H. Haskell†, R.B. Hayes†, V.V. Chumak* and G.H. Kenner \\ *Scientific Center of Radiation Medicine, Kiev, Ukraine; and †Center for Applied Dosimetry, University of \\ Utah, Salt Lake City, UT 84112
}

\begin{abstract}
The effect of the crushing and additive dose procedures used in EPR dosimetry of enamel was studied on the signals with $\mathrm{g}$-factors of 2.0045 and $\mathrm{g}_{1}=2.0018, \mathrm{~g}_{1}=1.9975$. Eight fractions, ranging in size from $<75$ micrometers to $2 \mathrm{~mm}$, were prepared from one tooth. Two cases were investigated: crushing of a non-irradiated sample and of a sample previously irradiated ( $6 \mathrm{~Gy}$ from ${ }^{\circ} \mathrm{Co}$ gamma ray source). In the non-irradiated study, the intensity of the native signal at 2.0045 increased by circa 1.75 times as the grain size decreased from maximum to minimum. A small decrease in radiation sensitivity $(<8 \%)$ was also observed with decreasing grain size. In the irradiated samples, crushing resulted in slight variations of reconstructed doses from expected values, but the worst possible case (grain sizes $<75 \mu \mathrm{m}$ ) showed that additional errors were less than $10 \%$.

The radiation sensitivity of enamel measured immediately after exposure is underestimated. It increases by about $15 \%$ in the first month. Based on the decomposition of the observed spectra, a new interpretation of transient signals is proposed which explains the above phenomena. Recommendations about how to use this interpretaion in retrospective EPR dosimetry are given.
\end{abstract}

\section{INTRODUCTION}

The First international intercomparison of EPR technique using tooth enamel (Chumak et al., 1996A) showed that the results obtained by different techniques may differ strongly especially at low doses, even with homogeneous enamel samples. One possible reason for this could be artifacts arising during sample preparation and irradiation procedures. The following steps are usually followed in evaluating radiation exposure.

\footnotetext{
* separation of enamel.

* preprocessing of enamel (crushing, chemical treatment, etc.).
} 
* irradiation.

* recording of EPR spectra.

* determination of intensity of radiation induced signal.

* determination of value of the accident dose absorbed in enamel by taking into account the components due to sample preparation, irradiation, environmental exposure and diagnostic $\mathrm{x}$-ray exposure.

Any of the above procedures may influence the results of dose reconstruction. It is known from the literature (Desrosiers et al., 1989, Iwasaki et al., 1993, Marino and Becker, 1968, Polyakov et al., 1995, Tatsumi-Miyajima and Okajima, 1985), that both the native EPR signal at $g=2.0045$ and the radiation-induced, anisotropic signal at $\mathrm{g}_{1}=2.0018, \mathrm{~g}_{1}=1.9975$ may change during crushing of enamel. It is also known (Oduwole and Sales, 1994), that additive laboratory irradiation generates short-lived EPR signals, which may influence the intensity of the radiation-induced signal.

The aim of this work was to investigate the influence of the enamel crushing and laboratory irradiation procedures, which are usually used in routine EPR-dosimetry. The parameters studied were intensity and radiation sensitivity of two of the main paramagnetic centers at $\mathrm{g}=2.0045$ and $\mathrm{g}_{\perp}=2.0018, \mathrm{~g}_{\text {, }}$ $=1.9975$. In relation to crushing effects, this work continues the previous investigation done by Polyakov et al. (1995) and Haskell et al. (1996). Here we have extended the range of grain size fractions, and applied the subtraction method used by the SCRM (Scientific Center of Radiation Medicine AMS Ukraine), for analysis of spectra (Chumak et al., 1996B). In addition, we evaluated both unirradiated and irradiated samples.

Relative to laboratory irradiation, we changed the doses used by Odowole and Sales (1994), to those actually used in EPR dosimetry. Also, we investigated the details of the transient EPR signals by varying the microwave power. This results in a new interpretation of the observed EPR signals in enamel close to $g=2.0$. Empirical coeffiecients were also established for correction of intensity of the signal at $g$ $=2.0018$, when measured soon after irradiation.

\section{MATERIALS AND METHODS}


Enamel for the experiments was obtained from routinely extracted teeth of patients less than 25 years-old at one of the student clinics in Kiev, Ukraine. The first step was the selection of samples for the crushing experiments. We decided to use enamel from only one tooth to avoid any influences of non-homogeneity of composition and properties. A very large tooth was chosen. The weight of pure enamel from this tooth was $675 \mathrm{mg}$. This tooth was designated as S1. The comparison of its EPR spectra with the spectra of other molars showed that it approximated the average intensity and shape of the native signal for grain sizes of $250-600 \mu \mathrm{m}$. In addition, it demonstrated average radiation sensitivity. The tooth was crushed into 4-6 big pieces using stomatological surgeon's pliars. The enamel was separated from the dentine by etching the pieces in $\mathrm{KOH}$ solution at $60^{\circ} \mathrm{C}$ in an ultrasonic bath (Branson 1210, Shelton, Conn). The $\mathrm{KOH}$ solution was periodically changed. The reaction was stopped after a few days of such treatment (the precise time depended on individual properties of teeth) and only pure enamel was left in the tube. By using this type of separation we obtained enamel which was not affected by crushing.

During chemical separation some of the big pieces broke up into smaller pieces. The separated enamel was divided into two parts: one part destined for crushing before irradiation with a weight of 339 $\mathrm{mg}$, and a second part, consisting of 19 large pieces with a combined weight $336 \mathrm{mg}$ and average size of 2-3 $\mathrm{mm}$, destined for irradiation after crushing. Thus, we considered this part as free from mechanicallystimulated signals.

The part destined for crushing before irradiation was crushed using a mortar and pestle into the following fractions, $600-850,425-600,250-425,150-250,106-150,75-106$ and $<75 \mu \mathrm{m}$ (the corresponding aliquots were designated as S1-1 to S1-7). The second part was crushed into the same fractions following irradiation (S1-8 to S1-14). Table 1 shows the characteristics of the aliquots.

The samples for investigating transient signals were obtained from a mixture of enamel from several teeth. The $250-600 \mu \mathrm{m}$ fraction was used following crushing with a mortar and pestle. The total weight of the enamel was $1350 \mathrm{mg}$, it was divided into 17 aliquots of $76-78 \mathrm{mg}$ each. The aliquots were labeled as $\operatorname{Tr} 1-\operatorname{Tr} 17$ and divided into pairs $\operatorname{Tr} 1-\operatorname{Tr} 2, \operatorname{Tr} 3-\operatorname{Tr} 4$, and iradiated withdifferent doses. The sample $\operatorname{Tr} 17$ was used as a native signal standard. 
The specimens were irradiated with using a Co- 60 source with a dose rate of $0.80 \mathrm{~Gy} / \mathrm{min}$ (Isotope Product Laboratory, Burbank, CA 91504). The dose of $6 \mathrm{~Gy}$ was administered to the second part of sample S1 as an analog of some "unknown" accident dose.

Seven additional exposures of 2 Gy each were done to determine the radiation sensitivity of individual aliquots ( Chumak et al., 1995). This exposure and corresponding EPR scanning was done on all aliquots of sample $S 1$ on the same day. This reduced possible errors due to daily variations in EPR spectrometer performance. The Tr1-Tr16 aliquots were irradiated with $0.5 \mathrm{~Gy}-100 \mathrm{~Gy}$. One of each pair was annealed at $95 \mathrm{C}$ for 2 hours, while the other was used to observe daily changes in the intensity of the EPR signals at ambient temperature.

Recording of EPR spectra was done using an $x$-band EPR spectrometer (Bruker, model ESP300E). If not stated otherwise, the following parameters were used, microwave power $10 \mathrm{~mW}$, modulation frequency $100 \mathrm{kHz}$, modulation amplitude $0.4 \mathrm{mT}$, sweep width $10 \mathrm{mT}$, time constant $20 \mathrm{~ms}$, conversion time $20 \mathrm{~ms}, 30$ to 60 sweeps. Calibration of $\mathrm{g}$-factor values was done using the third and fourth lines of a $\mathrm{Mn} 2+: \mathrm{MgO}$ standard continuously placed in the resonator.

The intensity of the $\mathrm{g}_{1}$ EPR signals were measured peak-to-peak after corrections were made on the original spectra which included subtraction of an empty tube spectra, recorded during the same day and with the same parameters as the sample (exceptions were made for high doses and low power where the influence of resonator baseline instability was negligible). An addtional subtraction of the native signal was done during measurement of the radiation induced signal. In this case, the maxima of the native signal of the standard spectra is adjusted until it is the same as the maxima of the native signal of the the radiation induced spectrum.

\section{RESULTS AND DISCUSSION}

\subsection{Crushing experiment.}

Crushing of non-irradiated samples mainly results in a dependence of native signal intensity on grain size (Fig. 1). We can see that intensity of the native signal has a tendency to monotonically increase within the investigated size range from 1 , that corresponds to signal in big grains, up to 1.75 , that 
corresponds to signal in the grains with size less than $75 \mu \mathrm{m}$. The slight increase in intensity of grains of size $600-850$ is probably without significance since later rechecking of the spectra showed increased spectrometer noise during the recording of these spectra. The dependency shown in Fig. 1 is probably due to generation of additional centers on the surface of sample during the crushing procedure. It is clear also from this dependency, that the quantity of additional centers generated during crushing is not linearly proportional to the increase of total surface area of the sample during this procedure, but has slight dependence on the characteristic size. We tried to estimate this empirically. The best extrapolation corresponds to a dependency between intensity of signal and mean of characteristic size in the following manner:

Intensity $1 /$ Intensity $2=(\text { Size2/Size } 1)^{1 / 2}$

in the size range $\sim 100$ to $300 \mu \mathrm{m}$. Above $300 \mu \mathrm{m}$ the native signal intensity does not depend on size, while for values less, than $100 \mu \mathrm{m}$ we do not have enough experimental data. This behavior of the native signal intensity probably relates to the composite multilevel volume arrangement of enamel, which results in only those surface centers which have orientation along the prism axis becoming paramagnetic.

No other major effects of crushing on non-irradiated samples were observed. The spectra of samples with smaller grain sizes frequently have additional low-intensity EPR signals, but these signals usually possess random properties, and they may be removed by additional purification of the sample, including heavy liquid separation and base-treatment in an ultrasonic bath.

Table 2 shows the effects of crushing on the sensitivity of irradiated specimens. In evaluating this data, allowance must be made for the small size of the possible effects and the influence of the finite, but reproducible measurement errors. In our estimation, based on the reproducibility of results with unirradiated spectra, these errors lie within $\sim 2 \%$. There is a small reduction in radiation sensitivity with decreasing sample size $(\sim 8 \%)$ of non-irradiated enamel (column 1$)$. Also seen is a small increase in radiation sensitivity of pre-irradiated compared to non-pre-irradiated specimens (column 1 vs. column 2). 
It is possible to explain this qualitatively by assuming, that only the center at $g_{1}=2.0018, g_{1}=$ 1.9975, which is located in the volume of the sample (Callens et al., 1995; Kenner et al., submitted) may become paramagnetic due to gamma irradiation. By decreasing the size of grains, we increase the total surface of sample and therefore the numbers of pre-centers, which are placed within the thin surface layer. Using this model we can estimate the thickness of the surface layer, whose pre-centers do not become paramagnetic with gamma irradiation. For this we use the fact that radiation sensitivity (which is proportional to the number of paramagnetic centers) was recuced by $\sim 8 \%$ while the characteristic size was reduced by $\sim 18$ times (from 675 to $37.5 \mu \mathrm{m}$ ). If we suppose for simplicity that $N$ is the total number of pre-centers which may become paramagnetic and designate $S$ as the total surface of sample with a mean size $675 \mu \mathrm{m}, \rho$ as the density of pre-centers and $\mathrm{h}$ as the thickness of the surface layer, within which the pre-centers are in the diamagnetic state, then for the EPR intensity of the radiation-induced centers for samples of 675 and $37.5 \mu \mathrm{m}$ respectively, we can write:

$I_{675}=A(N-S h \rho)$

$I_{375}=A(N-18 S h \rho)$

where A is a proportionality coefficient based on the quantity of paramagnetic centers and intensity of EPR signal. Equation 2 takes into account that the total surface of the sample is increasing versus its characteristic size if the mass is constant. Taking the ratios of equation 1 to equation 2 , and using the experimental value of $\sim 1.08$ for the ratio $\mathrm{I}_{\text {ors }}$ 137.s, $_{\text {, we obtain: }}$

$\mathrm{h}=0.0043 \mathrm{~N} / \mathrm{pS}$

Assuming that enamel grains are preferentially round spheres with radius $r=337.5 \mu \mathrm{m}$ and taking into account that $S=S_{i k}, N=V_{i} p k$, were $S_{i}$ and $V_{i}$ are the corresponding surface and volume of one grain and $k$ is the total number of grains in the sample, we can obtain the following value for $h$ : 
$\mathrm{h}=0.0043 \times \mathrm{\rho} / 3 \approx 0.5(\mu \mathrm{m})$

We can also explain the tendency for small increases in radiation sensitivity of samples exposed before crushing using the above model. It appears that pre-centers, once they become paramagnetic, remain in that state, even when they reach the surface h-layer. We decided to estimate the influence of this sensitivity change in samples imadiated before crushing on the accuracy of estimated doses. For this purpose, we reconstructed the "unknown" dose of 6 Gy using different grain size fractions. The results are shown in column 4 of Table 2 with the relative deviation of measured values shown in column 5 . An estimate of the accuracy of these measurements can be made by recognizing that all dose response curves of non-exposed, uncrushed specimens S1-1-. . . . $1-7$, excluding the one with a grain size of less than 75 $\mu \mathrm{m}$, intersected the dose-axes near the zero-point with deviations from zero of less than $\pm 50 \mathrm{mGy}$ (the S1-7 aliquot showed an initial dose of $340 \mathrm{mGy}$ ). We can see from Table 2 that in the worst case, when the fraction with a size of less than $75 \mu \mathrm{m}$ was used, the additional errors in dose determination due to influence of crushing on radiation sensitivity is $-10 \%$, and is much smaller in the case of fractions in the range $100-$ $850 \mu \mathrm{m}$.

\subsection{Experiment with transient signals.}

The results of the dose response study of samples with transient signals is shown in Fig. 2. The different lines correspond to the collection of EPR spectra at increasing time intervals. Curve 1 was measured one hour after exposure, curve 2 , six days and curve 3 , one month after exposure. The changes in sensitivity were: $6 \%$ after 6 days and $15 \%$ after one month. The last curve corresponds to saturation, as all transient signals relax by that time according to our data and that of Oduwole and Sales (1994).

We have also obtained the dose response curves for matched samples which were annealed at $95 \mathrm{C}$ for 2 hours immediately after exposure. The resulting curve was practically the same as curve 3 in Fig. 2 . Thus, there are two ways of removing transient signals in retrospective EPR-dosimetry. The first is to anneal the samples for 2 hours at a temperature of $90-95^{\circ} \mathrm{C}$ following exposure. The second is based on 
using empirical corrective coefficients. This is done as follows. The radiation induced signal intensity before subtraction is subtracted from the after exposure intensity. The resulting value is multiplied by a corrective coefficient based on time since exposure. If the spectra were measured on the same day, then this coefficient is equal c. 1.15. During the first week following exposure it decreases, by -0.1 per day. During the next two weeks by $\sim 0.1$ every two days. By three weeks after exposure the coefficient becomes equal to 1 .

Next we investigated the effect of microwave power on the transient signals. For this purpose the spectra of samples $\operatorname{Tr} 15$ and $\operatorname{Tr} 16$ were recorded using a wide range of microwave powers from a maximum of $200 \mathrm{~mW}$, attenuation $0 \mathrm{~dB}$, down to a few tens of $\mu \mathrm{W}$ in steps of $3 \mathrm{~dB}$. The original spectra of sample Tr15, corresponding to a measurement time of 3-4 hours after exposure, are shown in Fig. 3a. In the Fig. $3 b$ the same spectra are shown, but after subtraction of the corresponding native signals from sample Tr16 which had been annealed for two hours at $95^{\circ} \mathrm{C}$. Spectra of the $\operatorname{Tr} 15-\operatorname{Tr} 16$ samples were recorded under the same conditions during 2 hours on the same day, minimizing additional errors. The modulation amplitude used for registration of $\operatorname{Tr} 15-\operatorname{Tr} 16$ samples spectra was chosen at $0.2 \mathrm{mT}$ which corresponded to the width of the narrower transient signals. An additional adjustment was made for the magnitude of these spectra. Based on the data in Fig. 2, it was found that the differences in intensity of the $\mathrm{g}_{\mathrm{L}}=2.0018, \mathrm{~g}_{\mathrm{A}}=1.9975$ signals was such that the values of the Tr16 spectra were multiplied by 0.87 before subtraction even though the specimens had the same weight and received the same dose. The accuracy of this choice was shown by looking at the spectra taken at low microwave power (less than $0.1 \mathrm{~mW}$ ), where spectra shown consisted of only one transient signal after subtraction (Fig. $4 \mathrm{a}$, in which the resulting spectra corresponding to 0.0125 $\mathrm{mW}$ power is shown). The g-factor of this line and its width were 2.0034 and $0.24 \mathrm{mT}$ respectively which is in good agreement with data of Oduwole and Sales, (1994). With increasing microwave power the spectra evolve and become like the one shown in Fig. 4b, which corresponds to power $1.6 \mathrm{~mW}$. As before, we can see the signal with its center at $g=2.0034$ in the middle of the spectra, but now it is slightly degraded by other signals. An explanation of the spectra seen in Fig. $4 \mathrm{~b}$ could be that we have anadditional sextet at $\mathrm{g}=2.0030$ whose hyperfine splitting constant equals $\sim 0.6 \mathrm{mT}$. Only the first and second lines of this center can be seen in the original spectrum. We can see the 5th and 6 th lines after 
subtraction of the $g_{1}=2.0018$ and $g_{11}=1.9975$ signal, but the 3 rd and 4th lines are still masked by the signal at $g=2.0034$.

Using data from Fig. 3b, we plotted the power saturation curves for transient signal lines (Fig. 5). We can see that the signal from centers with $\mathrm{g}=2.0034$ is saturated at $\sim 1 \mathrm{~mW}$, while the intensity of the signal from line 1 of the assumed sextet, continues to increase up to maximum power. Taking into account that the signal from centers with $g=2.0034$ strongly affects the stable radiation induced signal, preference should be given for a power on the order of $10 \mathrm{~mW}$, at which the $\mathrm{g}=2.0034$ signal has saturated, but the intensity of the sextet is still small.

The question about the nature of transient signals is still open. Possibly, an experiment with using ENDOR may clear up whether we are dealing with 6 lines from one center or if the observed spectrum is a complicated superposition of lines from different centers. Anyway it is possible to say that observed transient signals shown in Fig. 3 are different from the native signal at $g=2.0045$, which usually dominates in the unexposed enamel sample. The latter signal differs in many parameters including $\mathrm{g}$-factor, width of line and power saturation curve from the corresponding parameters of transient signals.

\section{SUMMARY}

The experimental results provide possible explanations of the observed influence of the crushing procedures used in EPR dosimetry of tooth enamel on the paramagnetic centers at $\mathrm{g}=2.0045$ and $\mathrm{g}_{1}=2.0018, \mathrm{~g}_{11}=$ 1.9975. It was also shown that in the worst possible case, when we are using the fraction with grain size less than $75 \mu \mathrm{m}$ for dose reconstruction the effect of crushing results in a maximum additional error of $10 \%$. Detailed investigation of the properties of transient signals after gamma-ray exposure has suggested a new interpretation of their nature. The empirical corrective coefficients were determined which can be used to correct for the presence of transient signals.

Acknowledgements -- Supported by the U.S. Department of Energy, Contract DE-FC08-89NV10805 and U.S. Department of Energy by Lawrence Livermore National Laboratory under contract no. W-7405-ENG48. 


\section{REFERENCES}

Callens F, Moens P, Verbeeck R (1995) An EPR study of intact and powdered human tooth enamel dried at $400^{\circ} \mathrm{C}$. Calcif. Tissue Int. 56, 543-548.

Chumak V., Bailiff I., Baran N., Bougai A., Dubovsky S., Feedosov I., Finin V., Haskell E., Hayes R., Ivannikov A., Kenner G., Kirillov V., Khlamidova L., Kolesnik S., Liidja G., Likhtarev I., Lippmaa E., Maksimenko V., Meijer A., Minenko V., Pasalskaya L., Past J., Puskar J., Radchuk V., Sholom S., Skvortzov V., Stepanenko V., Vaher U., Wieser A. (1996A) The first international intercomparison of EPR dosimetry with teeth: first results. Appl. Radiat. Isot. 47(12).

Chumak V., Pavlenko Ju. and Sholom S. (1996B) An approach to the assesment of overall uncertainty of determination of dose using ESR technique. Appl. Radiat. Isot. 47(12).

Desrosiers M.F., Simic M.G. Eichmiller F.C., Johnston A.D. and Bowen R.L. (1989) Mechanicallyinduced generation of radicals in tooth enamel. Appl. Radiat. Isot. 40, 1195-1197.

Haskell E.H., Hayes R.B. and Kenner G.H. Preparation induced errors in EPR dosimetry of enamel: pre and post crushing sensitivity. Appl. Radiat. Isot. 47(12).

Iwasaki M., Miyazawa C., Uesawa T. and Niwa K. (1993) Effect of sample grain size on the CO33signal intensity in ESR dosimetry of human tooth enamel. Radioisotopes 42, 470-473.

Kenner G.H., Haskell E.H., Hayes R.B., Baig A. and Higuchi W.I. (submitted) ESR properties and dose response of hydroxyapatite and carbonated apatites. Calcif. Tissue Int.

Marino A.A. and Becker R.O. (1968) Mechanically induced free radicals in bone. Nature 218, 466-467.

Oduwole A.D. and Sales K.D. (1994) Transient ESR signals induced by g-irradiation in tooth enamel and in bone. Quaternary Geochronology 13, 647-650.

Polyakov V., Haskell E., Kenner G., Huett G. and Hayes R. (1995) Effect of mechanically induced background signal on EPR dosimetry of tooth enamel. Radiation Measurements 24, 249-254.

Tatsumi-Miyajima J. and Okajima S. (1985) ESR dosimetry using human tooth enamel. ESR Dating and Dosimetry (edited by M. Ikeya and T. Miki), IONICS, Tokyo), pp. 397-405. 
6.11 
Table 1. The original data characterizing aliquots of the sample S1. S1-1 to S1-7 were crushed before irradiation. S1-8 to S1-14 were irradiated post-crushing.

\begin{tabular}{|l|l|l|}
\hline Number of aliquot & Size of grains, $\mu \mathrm{m}$ & $\begin{array}{l}\text { Original weight of aliquot, } \\
\mathrm{mg}\end{array}$ \\
\hline S1-1 & $600-850$ & 47 \\
\hline S1-2 & $425-600$ & 49 \\
\hline S1-3 & $250-425$ & 50 \\
\hline S1-4 & $150-250$ & 50 \\
\hline S1-5 & $106-150$ & 35 \\
\hline S1-6 & $75-106$ & 39.5 \\
\hline S1-7 & less than 75 & 39 \\
\hline S1-8 & $600-850$ & 46 \\
\hline S1-9 & $425-600$ & 47 \\
\hline S1-10 & $250-425$ & 47 \\
\hline S1-11 & $150-250$ & 48.5 \\
\hline S1-12 & $106-150$ & 34 \\
\hline S1-13 & $75-106$ & 38.5 \\
\hline S1-14 & less than 75 & 40 \\
\hline
\end{tabular}


Table 2. Sensitivity of different grain size enamel samples corresponding to: 1) nonirradiated part of tooth (column 2), 2) part of tooth previously irradiated with dose of $6 \mathrm{~Gy}$. (column 3). Also shown are the results of the dose determination for each sample (column 4) and the relative deviation of the values in column 4 from the administered dose (column 5).

\begin{tabular}{|l|l|l|l|l|}
\hline $\begin{array}{l}\text { Range of grain } \\
\text { size, } \mu \mathrm{m}\end{array}$ & $\begin{array}{l}\text { Sensitivity of } \\
\text { enamel of } \\
\text { non-irradiated } \\
\text { part of tooth }\end{array}$ & $\begin{array}{l}\text { Sensitivity } \\
\text { of enamel } \\
\text { previously } \\
\text { irradiated } \\
\text { with dose } \\
\text { of 6 Gy }\end{array}$ & $\begin{array}{l}\text { Result of } \\
\text { determination of } \\
\text { dose 6 Gy using } \\
\text { different grain } \\
\text { size aliquots, } \\
\text { Gy }\end{array}$ & $\begin{array}{l}\text { Relative deviation } \\
\text { of previous } \\
\text { column data from } \\
\text { given dose, } \%\end{array}$ \\
\hline $600-850$ & 34.3 & 34.4 & 5.75 & -4.2 \\
\hline $425-600$ & 34.1 & 32.8 & 6.00 & 0 \\
\hline $250-425$ & 33.6 & 34.7 & 5.75 & -4.2 \\
\hline $150-250$ & 33.9 & 34.8 & 5.5 & -8.4 \\
\hline $106-150$ & 32.8 & 34.4 & 5.8 & -3.3 \\
\hline $75-106$ & 32.3 & 33.2 & 6.25 & +4.2 \\
\hline less 75 & 31.5 & 33.5 & 6.6 & +10 \\
\hline
\end{tabular}




\section{FIGURE CAPTIONS}

Fig. 1. Intensity of signal with $\mathrm{g}=2.0045$ in enamel as function of grain size.

Fig. 2. Measurements of dose response curves of enamel: 1 hour after irradiation (curve 1), 6 days after irradiation (curve 2) and 1 month after irradiation (curve 3).

Fig. 3. Spectra of sample with dose of $100 \mathrm{~Gy}$, recorded at different microwave powers. The upper spectra in parts $\mathrm{a}$ and $\mathrm{b}$ correspond to the maximum microwave power $200 \mathrm{~mW}$ (attenuation $0 \mathrm{~dB}$ ). The attenuation between each neighboring spectra is $3 \mathrm{~dB}$, so that the bottom spectra correspond to circa $0.0638 \mathrm{~mW}$ power ( $45 \mathrm{~dB}$ attenuation). Set a are the original spectra. Set $\mathrm{b}$ are the results of subtracting appropriate signals of sample Tr16 from the spectra of set a and consists of "pure" transient signals.

Fig. 4. Comparison of the responses of the paramagnetic centers at $g_{\perp}=2.0018, g_{n}=1.9975$ and $g=$ 2.0045 to sunlight and ultraviolet light respectively as a function of time. Curve $a$ is a plot of the intensity of the perpendicular component of th $\mathrm{g}_{1}=2.0018, \mathrm{~g}_{1}=1.9975$ signal in response to sunlight. The $y$-axis is expressed in terms of the amount of irradiation (mGy) required to obtain an equivalent response. Curve $b$ is the response of the signal at $g=2.0045$ to ultraviolet light and is normalized to the starting value.

Fig. 5. The power saturation curves of transient signals in the sample exposed to a dose of $100 \mathrm{~Gy}$. Line 1 is the saturation curve for signals with centers at $g=2.0034$. Line 2 is the saturation curve of the first component of the assumed sextet at $g=2.0030$ (low field maximum at $g=2.0124$ ). The inset is a magnified view of line 1 at lower powers. 


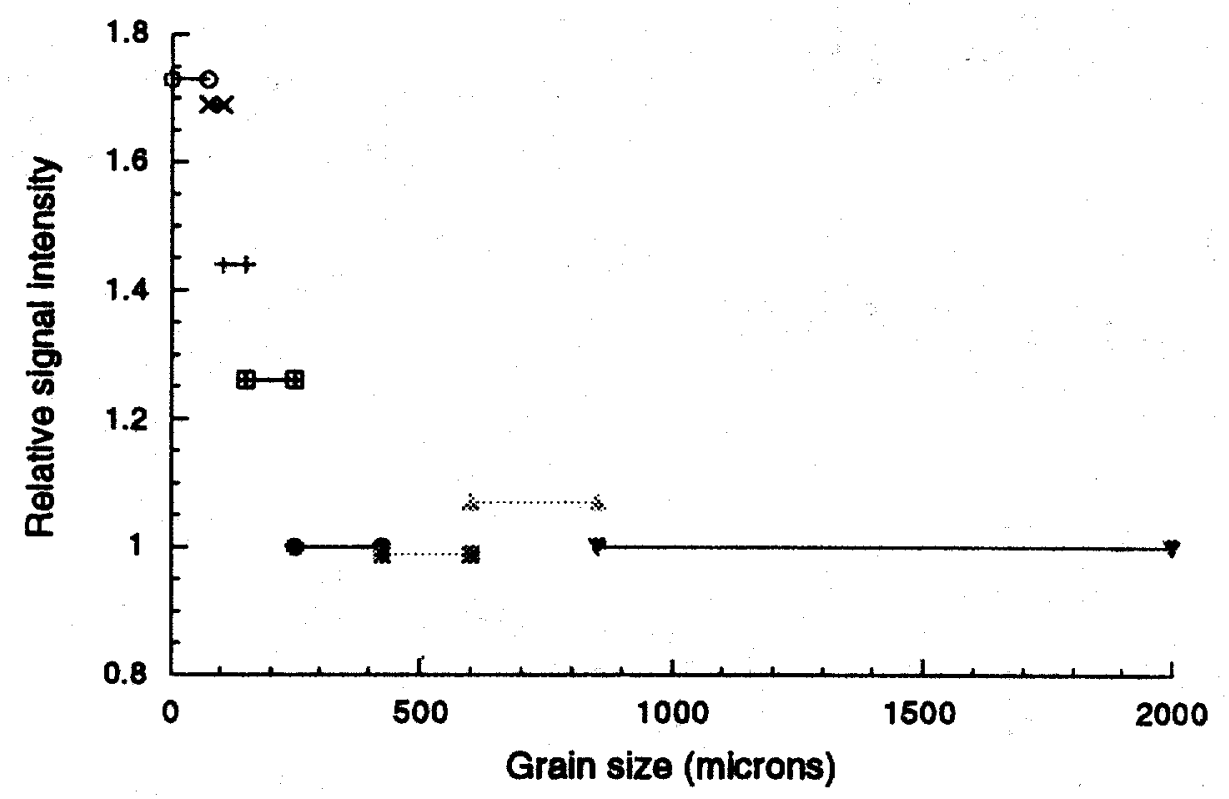




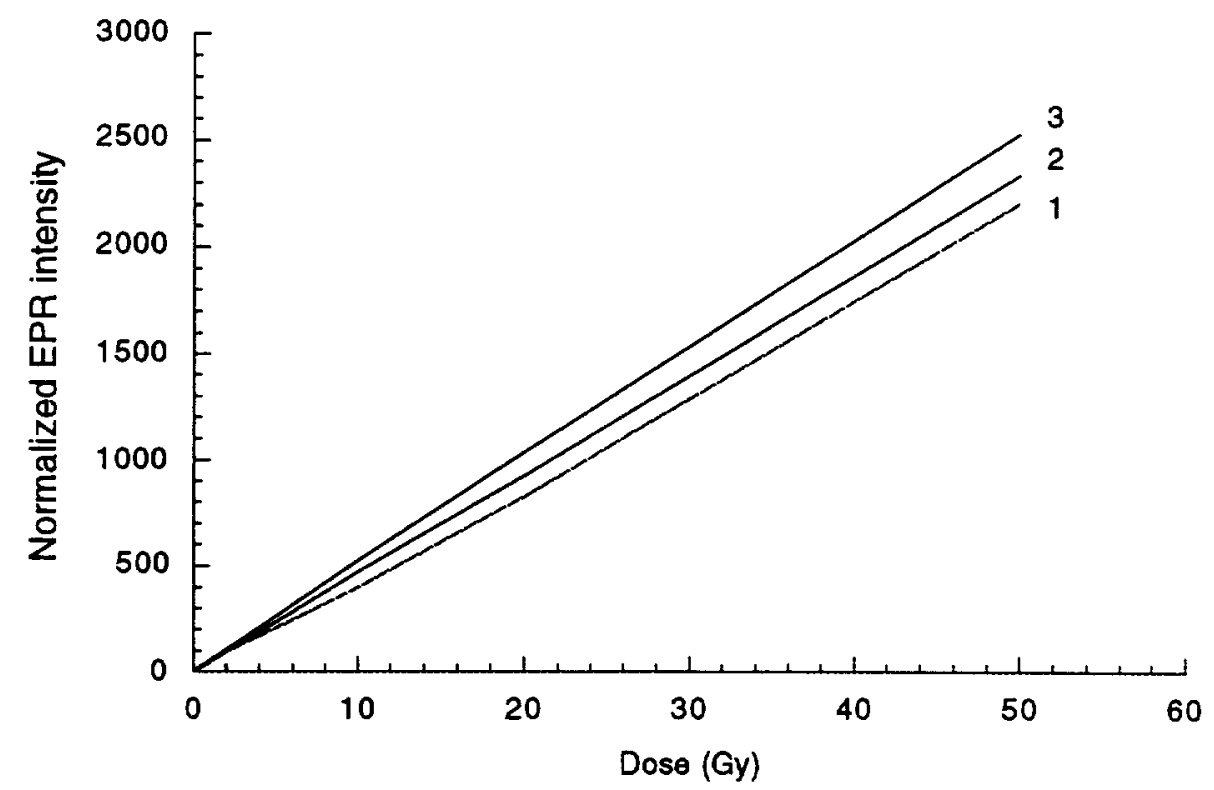



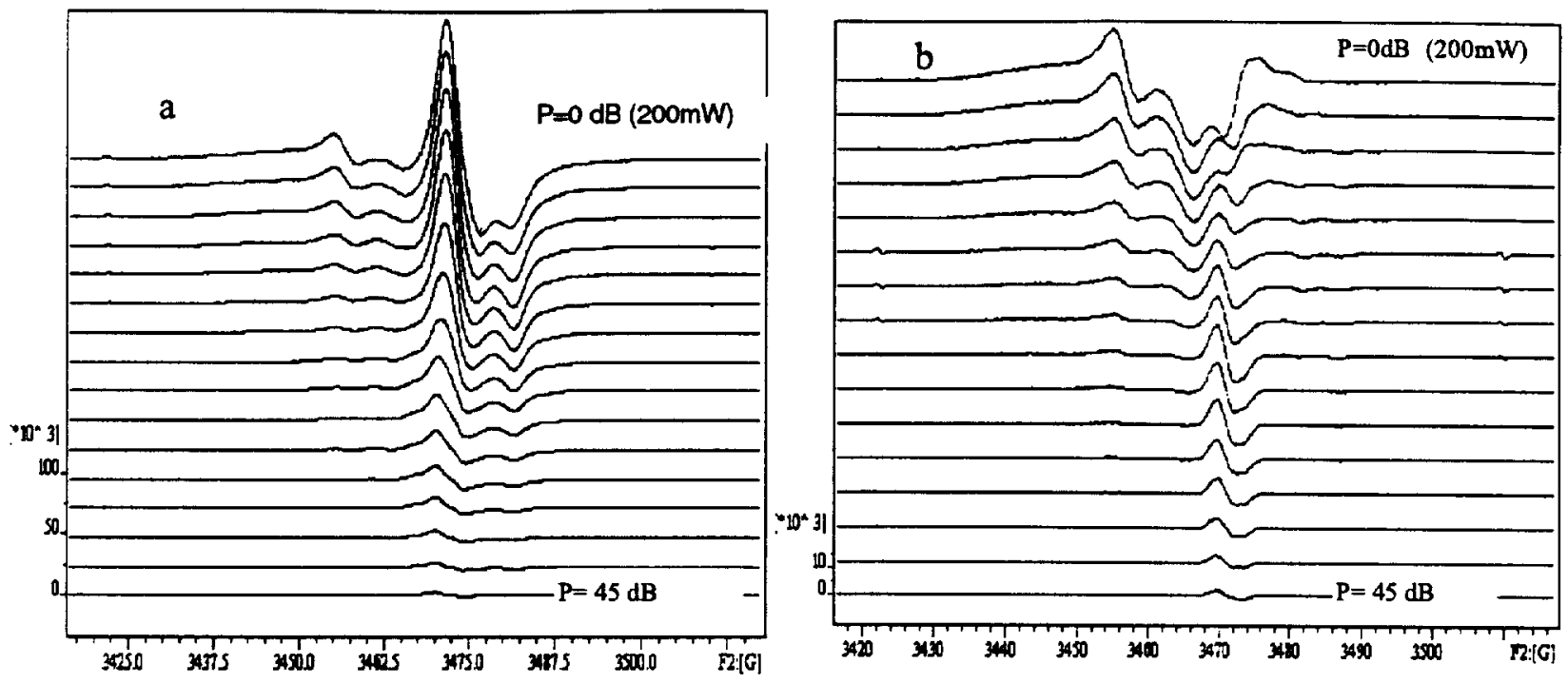

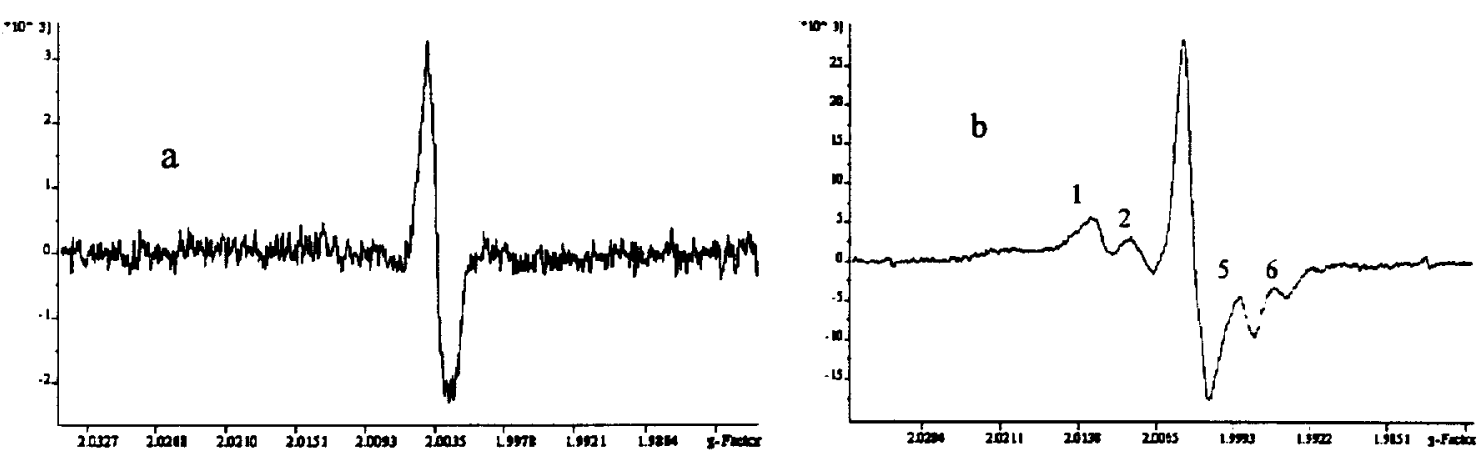


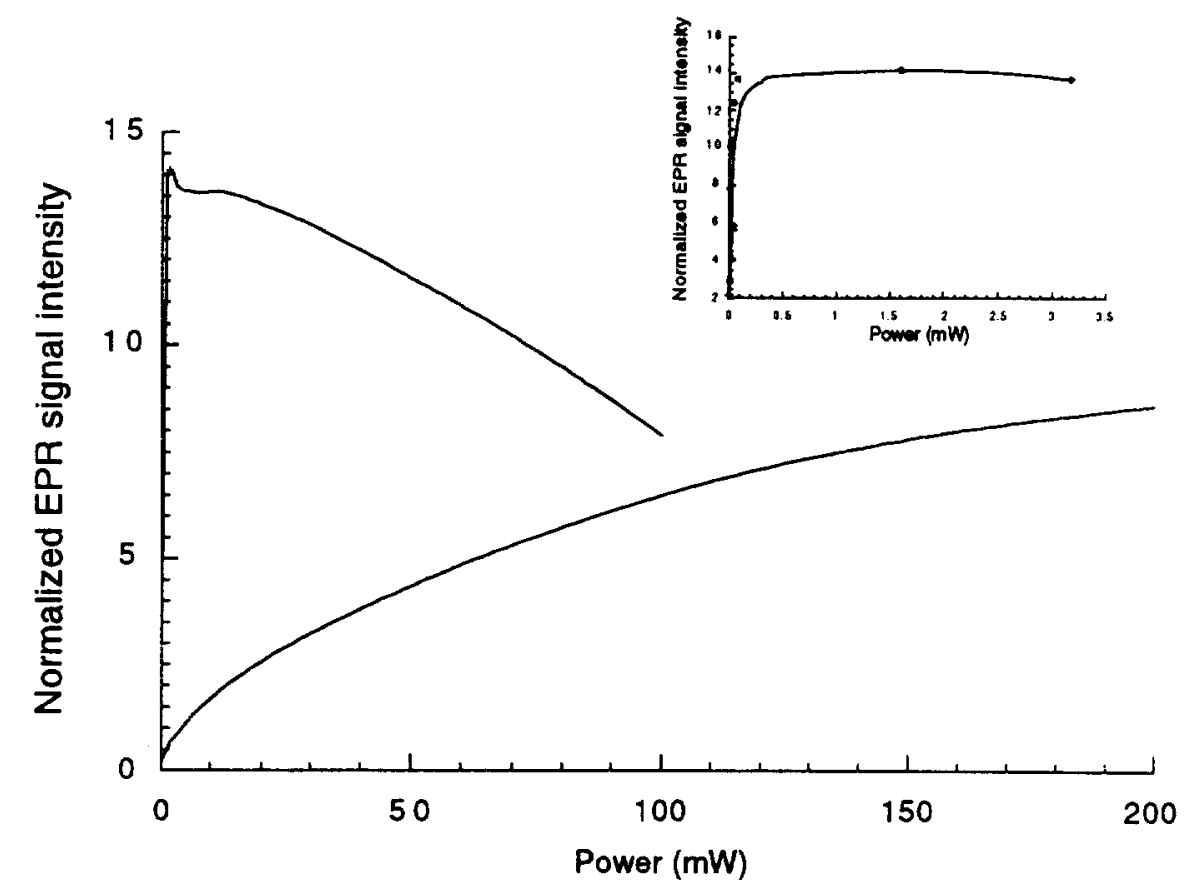

6.19 


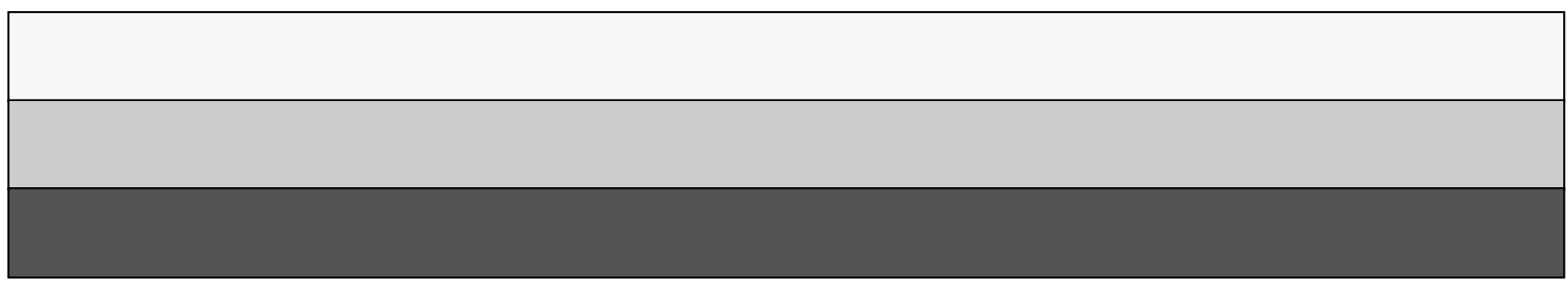

